\title{
Access to the brain parenchyma using endovascular techniques and a micro-working channel
}

\author{
Johan Lundberg, MD, PhD, ${ }^{1,2}$ Carina B. Johansson, PhD, ${ }^{3}$ Stefan Jonsson, $\mathrm{PhD},{ }^{4}$ and \\ Staffan Holmin, MD, PhD ${ }^{1,2}$
}

'Department of Clinical Neuroscience, Karolinska Institutet; 'Department of Neuroradiology, Karolinska University Hospital,

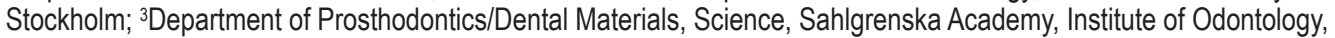
University of Gothenburg, Sweden; and "Department of Materials Science and Engineering, Royal Institute of Technology, Stockholm, Sweden

OBJECTIVE Several older studies report a low risk for parenchymal access to the CNS by surgical techniques. In more recent studies, including those with post-puncture CT scans, there are indications that the risk of bleeding might approach $8 \%$. New therapies, such as those that use viral vectors, modified mRNA, or cell transplantation, will probably warrant more parenchymal access to the CNS. Other minimally invasive routes might then be tempting to explore. This study was designed in 2 parts to address the possibility of using the endovascular route. The first aim was to test the ability to create a parenchymal micro-working channel to the CNS in macaque monkeys through the vessel wall. Second, the biocompatibility of a device-associated, detached, distal securing plug that was made of nitinol was investigated in swine for 1 year.

METHODS Trans-vessel wall intervention in the middle cerebral artery and associated cerebral parenchyma was performed in 4 rhesus macaque monkeys using a full clinical angiography suite. A contrast agent and methylene blue were injected to test the working channel and then detached at the distal end to act as a securing plug through the vessel wall. One-year follow-ups were also performed using angiography and histological analysis in 10 swine with 24 implants that were distributed in the external carotid artery tree.

RESULTS The cerebral interventions were performed without acute bleeding. Both the contrast agent and methylene blue were infused into the brain parenchyma and subarachnoidal space via the endovascular micro-working channel (7 injections in 4 animals). In the 1-year follow-up period, the implant that was left in the external carotid vessel wall in the swine was covered by the endothelium, which was followed by dislodgement just outside the blood vessel with thin capsule formation. No stenosis in the artery was detected on 1-year angiography. The animals showed normal behavior and blood sample results during the follow-up period. This is the first histological demonstration of nitinol biocompatibility when the implant is positioned through an arterial wall and indicates that the trans-vessel wall technique is not comparable with stent placement and its ability to induce intimal hyperplasia and restenosis.

CONCLUSIONS This study demonstrates that the trans-vessel wall technique is applicable to brain intervention in macaque monkeys, providing a micro-working channel for delivery or sampling. The long-term follow-up study of the detached device in swine showed no clinical or biochemical complications and a normal angiography appearance.

http://thejns.org/doi/abs/10.3171/2016.1.JNS152543

KEY WORDS endovascular; macaque; swine; minimal invasive; parenchymal puncture; nitinol; vascular disorders

$\mathrm{T}$ HE established minimally invasive methods for parenchymal access to the CNS currently include frameless or frame-based stereotactic techniques. Older studies indicated a very low risk of complications with the stereotactic technique. In a case series of 741 punctures in 500 patients, only 5 complications were re- corded: 2 intracranial hematomas, 1 infected free bone flap, 1 transiently increased neurological deficit, and 1 incident of a generalized epileptic event. ${ }^{1}$ Several therapeutic and diagnostic techniques currently require direct parenchymal access. However, more recent studies that include systematic imaging investigations indicate a higher 
risk for morbidity. Stereotactic brain biopsy carries a risk of hemorrhage that can be as high as $8 \%$ if routine postbiopsy CT scans are obtained. ${ }^{9}$ For other treatments that require parenchymal access, such as deep brain stimulation, the reported risk of hemorrhage varies considerably between $0 \%$ and $9.5 \% .^{2}$ Nowadays, it is possible to speculate that the indications for direct CNS parenchymal access might increase with the advent of new therapeutic regimes such as viral vectors, ${ }^{23}$ cell transplantations, ${ }^{3}$ modified mRNA, ${ }^{21}$ or other local treatments. With the increased demand for direct access, alternate routes for that access might be explored.

We previously described the use of a new endovascular catheter system used to access the parenchyma of hardto-reach organs (e.g., the pancreas) by creating a working channel from the femoral artery out through the vessel wall: the trans-vessel wall technique. ${ }^{17-19}$ We previously showed that it is possible to use a "nano-catheter" as the working channel through the vessel wall to deposit cells in the pancreas, and that the trans-vessel wall procedure in itself does not cause adverse events on 1-year angiographic follow-up. ${ }^{19}$ Furthermore, we have accessed the abdominal cavity and axillary pit ${ }^{17}$ as model systems for the technique.

Modern imaging-based endovascular interventional techniques now provide alternatives to open surgical access, and both arteries and veins can be regarded as "internal routes" to essentially anywhere in the body. A standard endovascular clinical catheter system, which includes an introducer, a guide catheter, and a microcatheter, is used to navigate within the vasculature to any place in the body. Once the microcatheter is in the desired location within the microvasculature, the prototype system is advanced through the microcatheter. The prototype catheter (outer diameter $0.193 \pm 0.0127 \mathrm{~mm}$, inner diameter 0.104 $\pm 0.0127 \mathrm{~mm}$, and total length $1700 \mathrm{~mm}$ ) then safely penetrates the arterial wall, as a nano-catheter, to reach the extravascular space (e.g., the parenchyma of the CNS) using the same principles as the introducer ${ }^{25}$ but with an inverted direction from inside the vessel toward the outside (Fig. 1). After this, we establish a working channel and have the ability to either administer substances or perform sampling. When the working channel is no longer in use, the most distal part can be detached and left in place as a securing plug in the vessel wall to prevent bleeding. This plug effectively results in a nitinol implant.

We previously concluded from studies in rats, rabbits, and swine that it is the intrinsic elastic effect of the vessel that prevents bleeding around the prototype catheter. ${ }^{18}$ We further concluded that the principle of the prototype catheter applies to vessel diameters around 0.5 to $1 \mathrm{~mm} .^{18}$ The rationale for the intrusion depth-limiting design is that even in high-magnification fluoroscopy, the resolution for optimal control is not sufficient. ${ }^{18}$ It is thus a safety precaution to avoid the risk for overshooting the system. The diameters are designed and verified by the loading cell tests. ${ }^{18}$ Furthermore, the inner diameter of the prototype system does not allow for bleeding since the driving pressure is the blood pressure, and by solving the Hagen-Poiseuille equation for this geometry, the flow will approach zero. This has also been confirmed in vivo in rats, rabbits, and swine. ${ }^{17-19}$

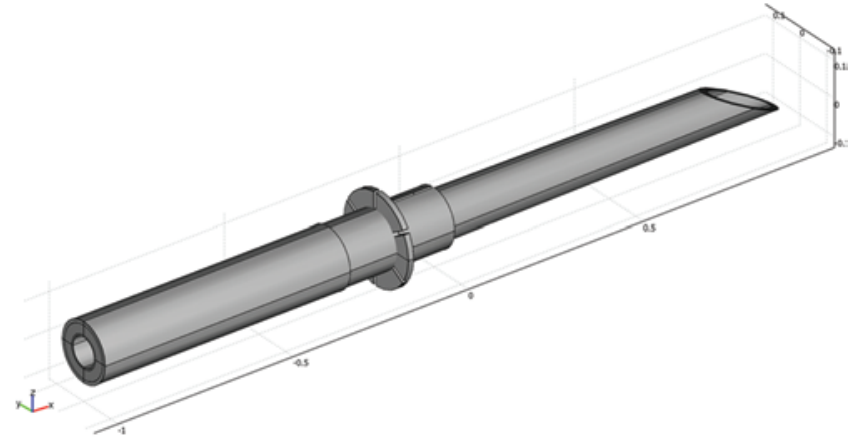

FIG. 1. A principal drawing of the most distal, sharp part of the design is depicted with an intrusion depth-limiting collar. Behind the depth-limiting collar, there is a detachment zone that is used to leave the distal end as the securing plug in the vessel wall. The physical dimensions of the entire catheter system are an outer diameter of $0.193 \pm 0.0127 \mathrm{~mm}$, an inner diameter of $0.104 \pm 0.0127 \mathrm{~mm}$, and a total length of 1700 $\mathrm{mm}$. This principal drawing shows the most distal $15 \mathrm{~mm}$. Reprinted from Lundberg J et al: PLOS ONE 5:e10449, 2010. doi:10.1371/journal. pone.0010449 under the Creative Commons Attribution License (http:// creativecommons.org/licenses/by/3.0/). Figure is available in color online only.

Nitinol (nickel-titanium) alloys are currently widely used because of their shape-memory and superelastic properties.7 The biocompatibility of nitinol has been thoroughly studied. ${ }^{4}$ It is well documented that the corrosion resistance of properly treated nitinol rivals that of titanium due to its ability to form a stable oxide layer on the surface that protects the bulk material. ${ }^{28,29}$ To improve the radio-opacity of nitinol implants placed under radiological guidance, radio markers are commonly attached, as, for instance, with stents. Galvanic corrosion must then be considered, and sufficient steps to hinder that must be taken. Nitinol and tantalum are galvanically similar and corrosion resistant. Gold and platinum are common radiopaque markers; however, they are both nobler than the nitinol alloy, which, unchecked, can cause severe galvanic corrosion. Hence, an insulating layer around the assembly has to be provided. ${ }^{8}$ One possible layer is parylene film, which also provides electrical insulation, excellent biocompatible properties, and is widely used in many medical devices and implants. ${ }^{27}$

The purpose of this 2-part study was first to investigate the use of the trans-vessel wall technique in the CNS vessels of the macaque brain and evaluate possible differences between intracerebral vasculature and our previous experience with extracerebral vasculature as a proof-ofconcept study without control groups. Second, we wanted to perform a histological and angiographic 1-year followup study on the detached distal tip in swine. In essence, this is a proof-of-concept study for this technique in the CNS setting.

\section{Methods}

All animal studies were conducted according to Karolinska Institutet guidelines for animal experiments. The studies were approved by the regional ethics committee for animal research in Stockholm, Sweden. 
Four rhesus macaques (Macaca mulatta) of Chinese origin, approximately 9 to 10 years old, were used in the experiment. They had been housed at the Astrid Fagraeus Laboratory animal facility at Karolinska Institutet, Stockholm, Sweden, for more than 6 years. Housing and care procedures were in compliance with the provisions and general guidelines of the Swedish Board of Agriculture, and the facility has been accredited by Association for Assessment and Accreditation of Laboratory Animal Care International. The animals were part of other long-term studies and planned for euthanization for medical reasons, and we applied to get permission to perform this study during the same anesthesia session that was planned for euthanization. All procedures were approved by the local ethics committee on animal experiments. The animals were housed in groups of up to 6 animals in combined indoor/outdoor pens with up to $20 \mathrm{~m}^{2}$ of floor area and a height of $2.5 \mathrm{~m}$. The pens were enriched to allow the animals to express their physiological and behavioral needs, and the animals were subjected to positive reinforcement training to reduce the stress associated with experimental procedures.

Ten Göttingen miniature swine were included in the long-term follow-up study. For this study, they received 1 or 2 implants each $(n=24)$ in the external carotid artery (ECA) as a simulation of the intracranial vasculature since the intracranial compartment in swine is not reachable via the endovascular route because of the rete mirabilis under the skull base. The swine were monitored with blood sampling until the follow-up angiographic examination was performed 1 year later. The same swine were used to harbor implants in the pancreas in previously published material. ${ }^{19}$

The trans-vessel wall prototypes were all manufactured by hand as previously described. ${ }^{18}$

\section{Macaque Anesthesia}

The sedation of the animals was initiated with the intramuscular injection of $10 \mathrm{mg} / \mathrm{kg}$ ketamine $(100 \mathrm{mg} /$ $\mathrm{ml}$ Ketaminol; Intervet), followed by sevoflurane (Baxter Medical AB) for both intubation and surgical anesthesia. A Siemens 900 servo-ventilator was used (Siemens Healthcare).

\section{Swine Anesthesia}

The induction of surgical anesthesia in the swine started with the intramuscular injection of $1 \mathrm{mg} / \mathrm{ml}$ Domitor (Orion BioPharma Animal Health) and $0.5 \mathrm{mg} / \mathrm{ml}$ atropine (Mylan $\mathrm{AB}$ ). The continuous infusion of pentobarbital and fentanyl $(50 \mu \mathrm{g} / \mathrm{ml}$; B Braun Medical) was used with a Siemens 900 servo-ventilator (Siemens Healthcare).

\section{Angiography}

Angiography was performed using the XD20 angiographic system and Philips 3DRA workstation (both Philips Medical System). The Visipaque 270 contrast agent (GE Healthcare) was used for all contrast-enhanced applications. Nimodipine calcium channel blockers (Bayer $\mathrm{AB}$ ) were used for spasm relaxation. XperCT images were obtained and reviewed using the XD20 system's XperCT high-dose program and soft-tissue algorithms (Philips Medical Systems). ${ }^{26}$

In swine, we used a 5-Fr introducer with femoral cutdown access (Terumo) and a 5-Fr Cobra guiding catheter with a Renegade microcatheter (Boston Scientific), and a Transend platinum tip guidewire (Boston Scientific) was navigated via the ECA to the maxillary artery.

In macaques, we used a 5-Fr introducer with femoral cut-down access (Terumo), and a 4-Fr vertebral guide catheter (Merit Medical) was used to navigate to the internal carotid artery. There, we used both Renegade HI-FLO and Prowler Select Plus (Codman) microcatheters with the Transend platinum tip guidewire.

The guidewire was withdrawn from the microcatheter, and the prototype catheter was deployed through the arterial wall. After finishing the procedure, a $10-\mathrm{V}$ current was applied, thereby detaching the distal part and leaving it as an implant through the arterial wall to control possible bleeding from perforation. The perforations were followed for up to 3 hours using high-resolution digital subtraction angiography. Following euthanasia with an overdose of pentobarbital, dissections were performed using an OPMI6-DF operating microscope (Carl Zeiss AB).

\section{Sample Preparation for Qualitative Histological Analysis}

The samples were retrieved with the implants in situ and immersed in a $4 \%$ neutral-buffered formaldehyde fixative and further processed in the laboratory to cut and grind the sections according to the method of Donath and Breuner. ${ }^{6}$ Upon arrival in the laboratory, each sample was radiographed to localize the device, which was then followed by trimming the specimen. In brief, the sample handling involved rinsing with water, dehydration in ethanol (from $70 \%$ to $99 \%$ ), pre-infiltration in diluted resin, and finally infiltration and embedding in pure resin and polymerized in ultraviolet light. ${ }^{13}$ The cured block was divided using a diamond band in a modified bandsaw during water cooling along the long axis of the needle device. The sample surface was ground in an orientation by placing the implant parallel to the grinding board and glued to Plexiglass sheet that served as the supporting object-glass. An initial thick section of about $150 \mu \mathrm{m}$ was prepared. The section was further ground using an automatic grinder and silicon carbide-water grinding papers of varying degrees of roughness. The final surface finish was prepared with smooth papers (2400 and 4000 grit). No further polishing was done.

The sections were histologically stained in toluidine blue mixed with pyronin $\mathrm{G}$, and they were coverslipped after drying. Light microscopic investigations were performed.

\section{Results}

We started the macaque experiments in a full clinical angiography suite. No problems were encountered with regard to anesthetizing the animals, and the femoral artery could easily be found using the cut-down technique. Initially, we navigated to the initial ascending intrapetrous segment of the internal carotid artery using a somewhat larger catheter system: the Renegade HI-FLO catheter that we previously used in the abdominal applications. Howev- 
er, in these subjects, the initial ascending intrapetrous segment did not permit navigation with the 2.8-Fr Renegade HI-FLO catheter, and hence a somewhat smaller 2.3-Fr Prowler Select Plus catheter was used instead. We could then navigate to the $\mathrm{M}_{2}-\mathrm{M}_{3}$ segment without being occlusive with the microcatheter. We also already noted in the cervical segment that the cerebral arteries of the animals were prone to spasm only by navigation and decided to add nimodipine calcium channel blockers to the pressurized infusions of the microcatheters that were already in place in the first subject.

After navigating to the target area, we changed the microwire to the trans-vessel wall device and established a working channel to the brain parenchyma $(n=7)$ (Fig. 2). No acute bleeding was observed on angiography through the microcatheter or from the guide catheter. We then proceeded to administer a contrast agent to visualize the parenchymal spread of administration, followed by methylene blue for necropsy evaluation (Fig. 2). All devices permitted intraparenchymal administration.

After administering fluids through the working channel, we detached the distal tip by applying a $10-\mathrm{V}$ direct current that was cycled through at 10 seconds on and 10 seconds off. This firmly detached all tips within 2 minutes of applying the cumulative direct current. After detachment, frontal, lateral, and oblique angiographic images were obtained. No bleeding, stenosis, or distal embolization could be visualized (Fig. 2). Signs of spasm could initially be observed that regressed following exposure with the nimodipine-mixed infusate through the microcatheter.

After each detachment, we obtained XperCT images of the detached distal tip. The injected contrast agent could easily be detected next to all detached devices, and the total occupying volume could be estimated (Fig. 2). Furthermore, no bleeding or ischemic lesions could be detected with the XperCT technique. We obtained 3D prop scans to further study the possible impact on the vessel tree. No pathological changes could be observed.

After completing the interventions, the animal was killed and the brain was removed. We could easily observe the deposited methylene blue in the parenchyma and also noted a slight tendency to spread in the subarachnoid space along the vessels of the "proto-insula" of the macaque (Fig. 2).

After removal of the brain, it was further dissected and photographed to evaluate any discrepancies between methylene blue and the contrast agent. As suspected, due to the properties of the 2 fluids, the methylene blue had spread further into the parenchyma.

Samples for histological analyses were taken with 1 sample, including the detached distal tip. This sample was then cut using the cutting-grinding technique. No microscopic evidence of burns or necrosis could be observed around the detached distal tip. Furthermore, histological investigations of the neurons showed no indication of stress from the downstream detachment.

One device in the series was selected to test the depthlimiting collar, and by applying a maximum pushing force on the microcatheter it was possible to displace the depthlimiting collar out through the vessel wall, which resulted in a limited subarachnoid hemorrhage.
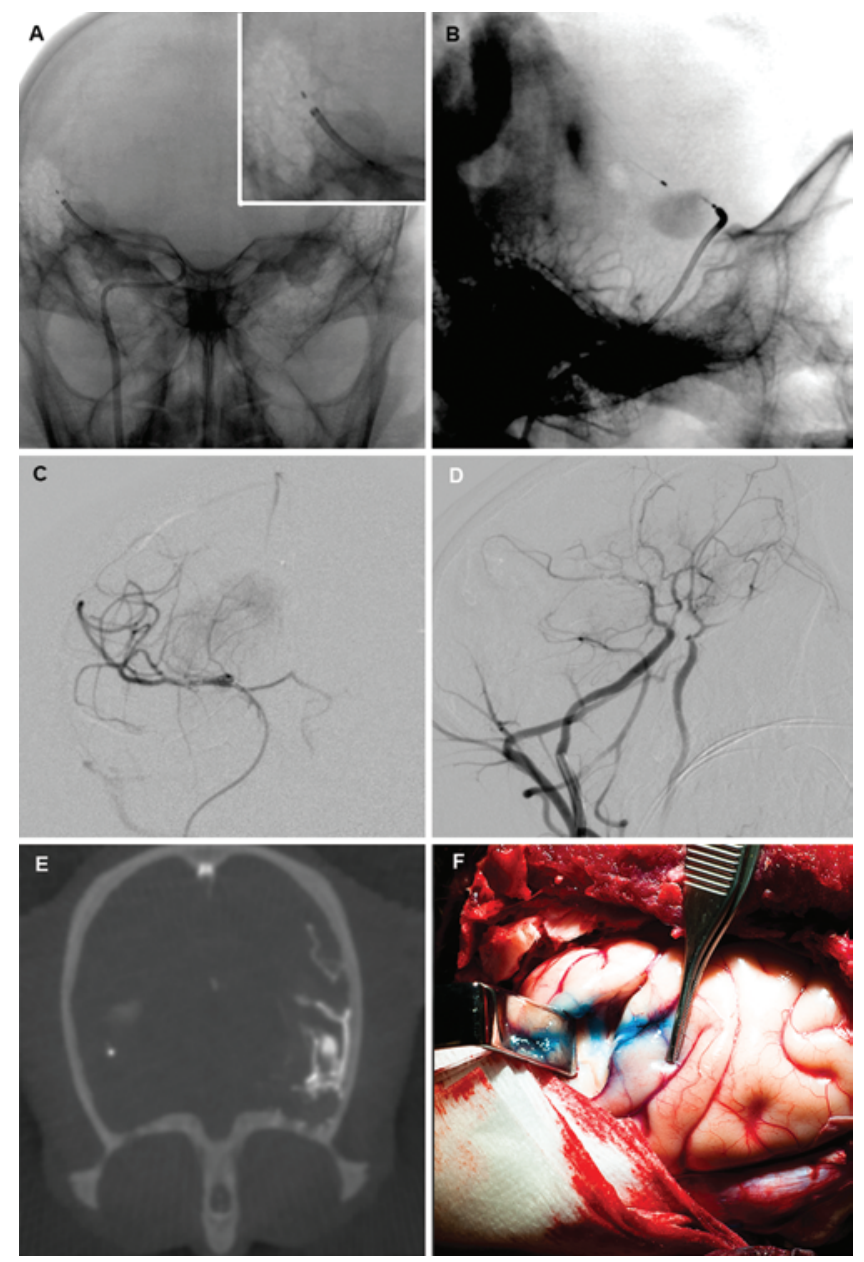

FIG. 2. A: A macaque cranium (posteroanterior view) showing the nanocatheter that has penetrated the vessel wall (inset). B: Lateral projection of another nano-catheter that is placed through the vessel wall with the intraparenchymal injection of the contrast agent. Note the previously detached distal end on the contralateral side shown in panel A. Posteroanterior (C) and lateral (D) view on digital subtraction angiography with detached distal tips. E: XperCT axial reconstruction with intraarterial contrast agent and the deposited extravascular contrast. F: Necropsy picture showing methylene blue deposited in the brain. Figure is available in color online only.

In the second part of the study, all 24 implants were positioned at the maxillary branch of the ECA in the swine; there were no instances of morbidity or mortality. Followup angiography at 1 year after implant insertion clearly showed that the implants had not in any way contributed to stenosis in the part of the vessel where the original intervention had been performed (Fig. 3). Furthermore, angiography and 3D angiography confirmed that all implants except $1(96 \%)$ had been dislodged from the bloodstream by approximately 0.5 to $1 \mathrm{~mm}$ (Fig. 3). This confirms the previous findings $\mathrm{s}^{19}$ with respect to angiography of the posterior pancreatic artery. In the ECA, all vessels were intact. As mentioned in Methods, the ECA implant site in the swine was chosen to mimic intracerebral implantation.

We used the XperCT technique to investigate the anatomical properties of the entire skull and tissues down- 
stream of the intervention site. We could observe neither cyst formation nor signs of embolism or other pathological conditions in the pterygopalatine fossa or elsewhere in the extracranial portions of the skull (Fig. 3).

The histological analysis was performed on the stained cut-and-ground sections with the implant in situ using a light microscope. The first finding was that the parylene coating was entirely intact in the devices themselves (Fig. 3). Furthermore, the devices showed no signs of galvanic corrosion on the interface between the nobler gold radiopaque marker and nitinol. This was observed uniformly in all implanted devices. Histological examination also confirmed that all implants (except 1) were dislodged from the bloodstream and the vessel itself for 0.5 to $1 \mathrm{~mm}$ (Fig. 3).

In all observed vessel walls adjacent to the implanted devices, the endothelium was normal. One implant within the ECA tree had not migrated through the endothelium and as such provided an interesting insight into implantendothelium interactions (Fig. 3). There was endothelial overgrowth of the most proximal part that, after detachment, was protruding into the lumen of the vessel. The endothelial coating was approximately $50 \mu \mathrm{m}$ wide at its widest portions. This endothelial coating was thin and thus could not be visualized by angiography. In both vessels containing implants through the vessel wall and vessels with implants outside the vessel wall, we could not observe any hyperplasia of the tunica media or endothelium in any cases.

We observed, in general, that the fibrous capsules that formed around the implants were all fairly well organized and especially apparent around the most distal part of the implant (Fig. 3). There was a large variability in the capsule radii, both along the long axis of the implants and between implants. The capsule was never thicker than 200 $\mu \mathrm{m}$. The surrounding tissue comprising muscle, fibrous tissue, nerves, and vessels all showed normal morphology without necrosis or signs of inflammation.

\section{Discussion}

From the first arterial-venous malformation embolization in the CNS that was reported in $1960^{16,20}$ and the advent of the digital subtraction angiography, ${ }^{22}$ the field of interventional neuroradiology has expanded rapidly. With new endovascular catheters, such as Guglielmi detachable coil $^{11,12}$ for aneurysm treatment and recent solid evidence for mechanical thrombectomy, ${ }_{, 10,14,24}$ the field of endovascular treatments is rapidly expanding. With this study, we want to further the reach of the possible endovascular therapies to also encompass the brain parenchyma itself.

The trans-vessel wall technique is primarily constructed for use against "hard-to-reach" organs, and in this study we present findings from the first application to the CNS. There are some important differences in the vascular anatomy in the CNS with respect to a relatively thinner wall in relation to its caliber. Furthermore, the internal elastica is more pronounced with fewer elastic fibers in the rest of the vascular wall, ${ }^{5}$ and therefore, as a proof of concept, it is important that we show that the trans-vessel wall technique can be applied within the CNS. The amount
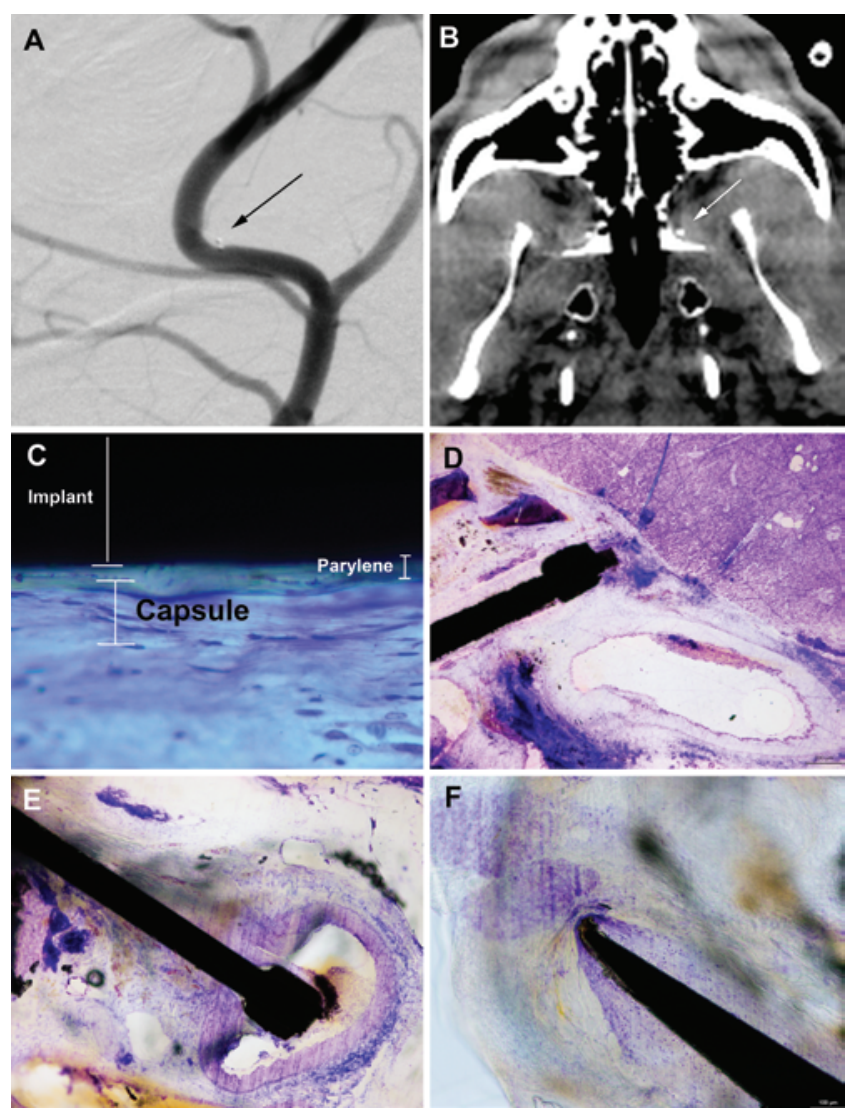

FIG. 3. A: Digital subtraction angiogram obtained 1 year after the transvessel wall technique, showing implantation with normal blood flow, no stenosis, and a dislodged distal tip due to contrast flow (arrow). B: XperCT axial reconstruction with the radiopaque marker indicated by the arrow in the pterygopalatine fossa. C: Extreme close-up of the implanted device, showing the parylene coating and the capsule histologically stained with toluidine blue mixed with pyronin G. D and E: The detached distal tip is shown (D), and only the tip is still in the vessel wall on follow-up (E). F: The tip of a detached distal tip with capsule formation. Figure is available in color online only.

of material used in this study is obviously too small to speculate on risk reduction as opposed to the reported, at maximum, $8 \%$ morbidity risk of direct parenchymal access through the skull bone. Nevertheless, as a proof of concept, we consider this small study interesting enough to merit further studies of the trans-vessel wall technique.

It is, for ethical reasons, difficult to perform long-term follow-up when testing a principally new technique, like this one, on nonhuman primates, and hence the long-term follow-up was performed in the swine extracranial vessels in order to study the biocompatibility of placing a nitinol device across the vessel wall. As in all experimental situations, there are trade-offs, and in swine it is that the rete mirabile hinders the navigation of catheters into the CNS. We considered the ECA tree of the swine to be a reasonable alternative to CNS placement.

The gold standard for evaluating implants residing in the vascular tree is angiography. This standard refers primarily to stents, and, as such, these guidelines might not be fully applicable to the new concept of trans-vessel wall 
nitinol implants. An important difference between stents and the detached distal tip lies in the design itself. Stents have a tubular design that supports the vessel wall and is known to incur restenosis..$^{15}$ We speculate that the natural rigidity from the tubular design makes endothelial cells grow over the stents, and they cannot be dislodged, thus resulting in restenosis. In the present study, angiography showed no stenosis formation of any kind in the ECA tree, hence resulting in the excellent vascular biocompatibility of this conceptual technique. This confirms previous findings ${ }^{19}$ with respect to angiography of the posterior pancreatic artery. We also performed XperCT to assess the global macroscopic tissue impact of the implants, readily showing no pathological processes and thereby providing solid safety data from a radiological perspective.

Furthermore, from the point of view of side effects, it is interesting that the implants seem to have been dislodged through the vessel wall. We speculate that the endothelium reorganizes and covers the implant, and since the implant is not tubular it can be dislodged by blood pressure during a long period of time and thus hinder bleeding. In a previous study in rabbits, a minority of the implants were found outside the vessel wall after 80 days. ${ }^{17}$ These implants would all have likely migrated had they been allowed to stay there for 1 year, as in this study.

The choice to perform the cutting-grinding technique with the implants in situ was made since previous findings ${ }^{17}$ and preliminary work prior to that showed that it is virtually impossible to study the implant-tissue interface without the implants being left behind as a marker. Further, the removal of implants of this size severely diminishes the evaluation of the small capsule formation (unpublished results). A possible trade-off is that the possible amount of capsule histology that can be analyzed becomes limited due to the properties of the cutting-grinding technique. The visual appearance of the capsule, which is fairly devoid of cells, and the "tautness" around the most distal part could possibly indicate that a shift from TNF- $\alpha$ to TGF- $\beta$ has occurred, thus rendering the capsule as nonprogressive. The relatively large variability in capsule thickness was also in line with previous findings. ${ }^{4}$ Even so, this variability was on a very small scale of capsule formation, thereby confirming that the trans-vessel wall implant is a fully reasonable implant for human studies.

\section{Conclusions}

We conclude from the first part of this study that the trans-vessel wall method is applicable to the CNS of the macaque. It can probably be used for a wide variety of indications that require CNS parenchymal access. Further preclinical testing (e.g., using MRI) is warranted prior to clinical Phase I testing with respect to safety and the risk of ischemic events.

From the second part of the study, we conclude that at 1 year after implantation endothelial cells grow over the vast majority of implants, and then the majority become dislodged out of the bloodstream during the rebuilding of the vasculature architecture. Thereafter, the implants become encapsulated and a very limited inflammatory response can be observed in both the pancreas and within the ECA tree. This would indicate that the trans-vessel wall technique is not comparable with stent placement and its ability to induce restenosis.

\section{Acknowledgments}

We thank Johanna Doshé, Gudrun Nylén, and Petra Hammarström Johansson for providing excellent technical assistance. This study was supported by grants from the Karolinska Institutet funds, Karolinska Institutet Innovations AB, the Swedish Society of Medicine, Vinnova Vinn Verifiering, The Swedish Brain Fund, and Strokefonden. The funders had no role in study design, data collection and analysis, decision to publish, or preparation of the manuscript.

\section{References}

1. Apuzzo ML, Chandrasoma PT, Cohen D, Zee CS, Zelman V: Computed imaging stereotaxy: experience and perspective related to 500 procedures applied to brain masses. Neurosurgery 20:930-937, 1987

2. Ben-Haim S, Asaad WF, Gale JT, Eskandar EN: Risk factors for hemorrhage during microelectrode-guided deep brain stimulation and the introduction of an improved microelectrode design. Neurosurgery 64:754-763, 2009

3. Bliss T, Guzman R, Daadi M, Steinberg GK: Cell transplantation therapy for stroke. Stroke 38 (2 Suppl):817-826, 2007

4. Castleman LS, Motzkin SM, Alicandri FP, Bonawit VL: Biocompatibility of nitinol alloy as an implant material. J Biomed Mater Res 10:695-731, 1976

5. Cipolla MJ: Anatomy and ultrastructure, in The Cerebral Circulation. San Rafael, CA: Morgan \& Claypool Life Sciences, 2009

6. Donath K, Breuner G: A method for the study of undecalcified bones and teeth with attached soft tissues. The SägeSchliff (sawing and grinding) technique. J Oral Pathol 11:318-326, 1982

7. Dotter CT, Buschmann RW, McKinney MK, Rösch J: Transluminal expandable nitinol coil stent grafting: preliminary report. Radiology 147:259-260, 1983

8. Duda SH, Wiskirchen J, Tepe G, Bitzer M, Kaulich TW, Stoeckel D, et al: Physical properties of endovascular stents: an experimental comparison. J Vasc Interv Radiol 11:645654,2000

9. Field M, Witham TF, Flickinger JC, Kondziolka D, Lunsford LD: Comprehensive assessment of hemorrhage risks and outcomes after stereotactic brain biopsy. J Neurosurg 94:545-551, 2001

10. Goyal M, Demchuk AM, Menon BK, Eesa M, Rempel JL, Thornton J, et al: Randomized assessment of rapid endovascular treatment of ischemic stroke. N Engl J Med 372:10191030,2015

11. Guglielmi G, Viñuela F, Dion J, Duckwiler G: Electrothrombosis of saccular aneurysms via endovascular approach. Part 2: Preliminary clinical experience. J Neurosurg 75:8-14, 1991

12. Guglielmi G, Viñuela F, Sepetka I, Macellari V: Electrothrombosis of saccular aneurysms via endovascular approach. Part 1: Electrochemical basis, technique, and experimental results. J Neurosurg 75:1-7, 1991

13. Johansson CB, Morberg P: Importance of ground section thickness for reliable histomorphometrical results. Biomaterials 16:91-95, 1995

14. Jovin TG, Chamorro A, Cobo E, de Miquel MA, Molina CA, Rovira A, et al: Thrombectomy within 8 hours after symptom onset in ischemic stroke. N Engl J Med 372:2296-2306, 2015

15. Karas SP, Gravanis MB, Santoian EC, Robinson KA, Anderberg KA, King SB III: Coronary intimal proliferation after 
balloon injury and stenting in swine: an animal model of restenosis. J Am Coll Cardiol 20:467-474, 1992

16. Luessenhop AJ, Spence WT: Artificial embolization of cerebral arteries. Report of use in a case of arteriovenous malformation. JAMA 172:1153-1155, 1960

17. Lundberg J, Jonsson S, Holmin S: Long term follow-up of the endovascular trans-vessel wall technique for parenchymal access in rabbit with full clinical integration. PLoS One 6:e23328, 2011

18. Lundberg J, Jonsson S, Holmin S: New endovascular method for transvascular exit of arteries and veins: developed in simulator, in rat and in rabbit with full clinical integration. PLoS One 5:e10449, 2010

19. Lundberg J, Stone-Elander S, Zhang XM, Korsgren O, Jonsson S, Holmin S: Endovascular method for transplantation of insulin-producing cells to the pancreas parenchyma in swine. Am J Transplant 14:694-700, 2014

20. Maiti TK, Bir SC, Bollam P, Nanda A: Alfred J. Luessenhop and the dawn of a new superspecialty: endovascular neurosurgery. J Neurointerv Surg 8:216-220, 2016

21. McIvor RS: Therapeutic delivery of mRNA: the medium is the message. Mol Ther 19:822-823, 2011

22. Meaney T, Weinstein M, Buonocore E, Pavlicek W, Borkowski GP, Gallagher JH, et al: Digital subtraction angiography of the human cardiovascular system. AJR Am J Roentgenol 135:1153-1160, 1980

23. Rosenberg JB, Sondhi D, Rubin DG, Monette S, Chen A, Cram S, et al: Comparative efficacy and safety of multiple routes of direct CNS administration of adeno-associated virus gene transfer vector serotype rh.10 expressing the human arylsulfatase A cDNA to nonhuman primates. Hum Gene Ther Clin Dev 25:164-177, 2014

24. Saver JL, Goyal M, Bonafe A, Diener HC, Levy EI, Pereira VM, et al: Stent-retriever thrombectomy after intravenous t-PA vs. t-PA alone in stroke. N Engl J Med 372:2285-2295, 2015

25. Seldinger SI: Catheter replacement of the needle in percuta- neous arteriography; a new technique. Acta Radiol 39:368376,1953

26. Söderman M, Babic D, Homan R, Andersson T: 3D roadmap in neuroangiography: technique and clinical interest. Neuroradiology 47:735-740, 2005

27. Song JS, Lee S, Jung SH, Cha GC, Mun MS: Improved biocompatibility of parylene-C films prepared by chemical vapor deposition and the subsequent plasma treatment. J Appl Polym Sci 112:3677-3685, 2009

28. Wever DJ, Veldhuizen AG, de Vries J, Busscher HJ, Uges DR, van Horn JR: Electrochemical and surface characterization of a nickel-titanium alloy. Biomaterials 19:761-769, 1998

29. Wever DJ, Veldhuizen AG, Sanders MM, Schakenraad JM, van Horn JR: Cytotoxic, allergic and genotoxic activity of a nickel-titanium alloy. Biomaterials 18:1115-1120, 1997

\section{Disclosures}

The catheter system described in this paper is owned by Smartwise AB, and Drs. Lundberg, Jonsson, and Holmin report that they have financial interest in this company.

\section{Author Contributions}

Conception and design: Holmin, Lundberg. Acquisition of data: all authors. Analysis and interpretation of data: all authors. Drafting the article: Lundberg. Critically revising the article: all authors. Reviewed submitted version of manuscript: Holmin, Lundberg. Approved the final version of the manuscript on behalf of all authors: Holmin. Administrative/technical/material support: all authors. Study supervision: Holmin, Lundberg.

\section{Correspondence}

Staffan Holmin, Department of Neuroradiology, Karolinska University Hospital, Solna, Stockholm 171 76, Sweden. email: staffan.holmin@ki.se. 